\title{
Zero-Compression, Laminar Electron Beam Generation in a Uniform Magnetic Field
}

This paper was downloaded from TechRxiv (https://www.techrxiv.org).

\section{LICENSE}

CC BY 4.0

SUBMISSION DATE / POSTED DATE

23-06-2021 / 05-07-2021

CITATION

Jackson, Robert; Ives, R. L.; Read, M. E. (2021): Zero-Compression, Laminar Electron Beam Generation in a Uniform Magnetic Field. TechRxiv. Preprint. https://doi.org/10.36227/techrxiv.14832348.v1

$\mathrm{DOI}$

10.36227/techrxiv.14832348.v1 


\title{
Zero-Compression, Laminar Electron Beam Generation in a Uniform Magnetic Field
}

\author{
R. H. Jackson, Jr., Life Senior Member, R. L. Ives, Life Senior Member, and M. E. Read, Life Senior \\ Member
}

\begin{abstract}
The need for enhanced performance of highpower RF vacuum electron devices has led to investigation of multiple-beam, sheet beam and annular beam configurations. A key issue with such devices is the magnetic field shaping required to produce high-power, laminar beams. Field shaping is difficult when Pierce-type gun geometries are employed. The development of high current density cathodes makes the necessary beam power achievable without compression. Such cathodes can operate within a uniform magnetic field yielding advantages for both single and distributed-beam RF devices. However, the quality of the resulting beams presents problems. A project to optimize beam quality in zero-convergence electron guns was undertaken by Calabazas Creek Research (CCR) and North Carolina State University (NCSU). The surprising result was that high quality electron beams can be generated in uniform magnetic fields using convex (dome) shaped cathodes. The underlying physics involves perturbation of the beam cyclotron motion by a non-adiabatic radial electric field impulse. This paper examines this physical mechanism and extends the initial result to additional diode and beam geometries.
\end{abstract}

Index Terms-Diodes, Electron beams, Electron guns, Focusing.

\section{INTRODUCTION}

Development of zero convergence electron guns is driven by requirements for RF sources at higher frequencies and higher power [1]. As the frequency increases, it becomes difficult to match electrostatic fields with converging magnetic fields for beam compression. This leads to poor quality beams and reduced performance. Recent developments with Controlled Porosity Reservoir and scandate cathodes [2], [3], [4] allow cathode current densities exceeding 20-30 A/ $\mathrm{cm}^{2}$ without sacrificing lifetime. Such emission densities are sufficient to eliminate beam compression requirements for many applications. The desire to reduce beam voltage using multiple beam guns also drives the need for zero compression beams where high current density cathodes allow packing beams closer to the device axis, a requirement for higher frequency

R. H. Jackson, Jr. is a retired CCR senior scientist but continues to do research on topics of personal interest.; (e-mail: r.h.jackson@ieee.org).

R. L. Ives and M. E. Read are with Calabazas Creek Research, Inc., 690 Port Drive, San Mateo CA 94404, USA. devices. This reduces or eliminates the opportunity for beam compression in the gun.

In 2002, Calabazas Creek Research, Inc. (CCR) participated in a Research Experience for Undergraduates (REU) program at North Carolina State University (NCSU) [5]. CCR was involved in teaching undergraduate mathematics students the advantages of computer optimization for electron gun design. CCR's Beam Optics Analyzer (BOA) code [6] was utilized to design a multiple-beam electron gun for a $200 \mathrm{MHz}$ klystron. Because of the large beam size no compression was needed to achieve the required current density. It was hoped that a laminar beam could be achieved by optimizing Pierce gun geometric parameters and magnetic field strength.

In what is surely a classic example of "they were left unattended," the NCSU students 'incorrectly' set the constraints on the cathode radius of curvature. In the optimization process, BOA determined that the optimum geometry for a zero-compression, confined-flow beam was a dome shaped convex cathode as illustrated in Fig. 1. This result, the exact reverse of the standard geometry, was both unexpected and puzzling [7]. Further analysis revealed additional beneficial characteristics. The dome cathode configuration does not require a focus electrode, so electric field gradient issues are reduced. The dimensional tolerances on quantities such as focus electrode concentricity, radial spacing, and axial positioning of focusing components can be relaxed without significant impact on beam performance. Edge emission is virtually eliminated.

On the other hand, operation of a dome cathode gun involves space-charge limited emission at substantial angles to a uniform axial magnetic field, trajectory deflection by a radial electric impulse at the anode aperture and transition of the beam to equilibrium in the beam tunnel. The interplay between these processes to achieve a laminar beam will be examined in this paper. The next section will develop a parametric model of the equilibrium envelope of a rippled beam and use it to examine ripple dependencies. The third section presents detailed simulations of dome cathode gun operation and examines the characteristics of the generated beams. Section IV will focus on the internal characteristics of 


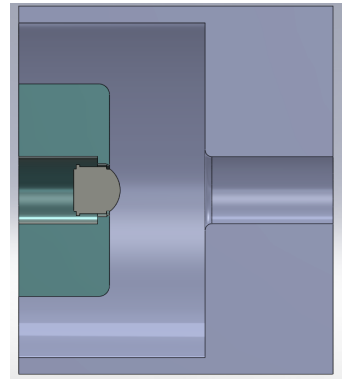

Fig. 1. Basic dome cathode geometry developed during the CCRNCSU Research Experience for Undergraduates program. Note that focusing elements are not required on either the cathode or anode plates. The flat profiles of both surfaces eliminates the geometric features causing high voltage breakdown.

dome-cathode beams. In Section V, the robustness and flexibility of the dome cathode configuration will be explored. The final section will summarize our results and discuss potential applications.

The use of non-adiabatic electric and magnetic fields to alter beam properties has been previously explored, see [11], [12] and references therein. In [11], an electron gun was fabricated with a non-adiabatic magnetic field in the diode. This gun was tested and measurements compared with simulation results. The final section will briefly discuss the work in [11], [12] and its relation to this paper.

A mix of $M K S, c g s$, numerical and normalized units are used for physical parameters, equations and figures. We have endeavored to clearly indicate the units used in each case.

\section{PARAmetric Modeling of BeAm RiPPle}

The key physical processes in the dome cathode geometry occur in three regions: 1) cathode dome, 2) anode aperture and 3) uniform beam tunnel. Because of their complexity, the first two regions will be investigated in the next section via simulations. The third region, the beam tunnel, is the least complex in terms of geometry and beam behavior, and will be examined in this section. The confined-flow beam envelope will be explored by developing an ordinary differential equation (ODE) model describing the behavior of a beam with laminar (non-crossing) cyclotron ripples.

Practical high-current beams require guiding magnetic fields if the beam propagates significant distances, e.g. multiple RF cavities or several sections of slow-wave structure. In such cases the magnetic field "threads" the cathode resulting in confined flow. In cylindrical configurations with static fields, the azimuthal equation of motion yields conservation of canonical angular momentum as shown in (1) where $C$ is a constant of integration, $A_{\theta}$ is the azimuthal vector potential component, $B_{z}$ is the axial magnetic field (uniform in this case) corresponding to $A_{\theta}, \beta_{i}=v_{i} / c, \eta=q /\left(\gamma_{o} m c^{2}\right)$ and $\gamma_{o}$ is the usual relativistic factor. (Equations 1 through 5 use $c g s$ units.)

$$
\beta_{\theta}=-\eta A_{\theta}+\frac{C}{r}=-\frac{1}{2} \eta r B_{z}+\frac{C}{r}
$$

In confined flow, $C$ has a value determined by the magnetic field at the emission point. This leads to the expression given in (2) where $r_{o}$ is the emission radius, electrons are assumed to have zero azimuthal velocity at emission and the magnetic field is uniform.

$$
\beta_{\theta}=\frac{1}{2} \eta r_{o} B_{z}\left(\frac{r_{o}}{r}-\frac{r}{r_{o}}\right)
$$

We will assume the beam is laminar (ripples but no ray crossings) and exhibits reasonably uniform density and axial velocity, see Fig. 5 in the next section. The self-generated fields at the beam edge can then be approximated by the expressions in (3).

$$
B_{\theta}(r)=\frac{2 I_{b}}{c r} \quad E_{r}(r)=\frac{2 I_{b}}{c r} \frac{1}{\beta_{z}}
$$

It will also be assumed that in the equilibrium state, the axial velocity and energy at a given average radius in the beam are roughly constant, $\dot{\beta}_{z} \simeq 0$ and $\gamma(z) \simeq \gamma_{o}$. These assumptions are supported by the simulation results presented in Section III. Hence, the only equation-ofmotion of concern is the radial equation, which can be written as shown in (4) where $\tau=c t$.

$$
\frac{d \beta_{r}}{d \tau}-\frac{1}{r} \beta_{\theta}^{2}=\eta\left[E_{r}+\beta_{\theta} B_{z}-\beta_{z} B_{\theta}\right]
$$

The radial electric field consists of two components, the beam self-field (3) and an applied field $E_{A}(z)$ defined below in (14).

$$
E_{r}=\frac{2 I_{b}}{c r} \frac{1}{\beta_{z}}+E_{A}
$$

Substituting (2), (3) and (5) into (4) and rearranging results in the expression below where $\lambda=\beta_{z} / \beta_{o}, B_{B}$ is the Brillouin magnetic field, $B_{z}=p B_{B}$ and $\beta_{o}=$ $\sqrt{\gamma_{o}^{2}-1} / \gamma_{o}$

$$
\begin{gathered}
\frac{d^{2} r}{d \tau^{2}}=r_{o} \mu^{2}\left[\left(\frac{r_{o}^{3}}{r^{3}}-\frac{r}{r_{o}}\right)+\sigma \frac{r_{o}}{r}\right]+\eta E_{A} \\
\mu=\frac{1}{2} \eta p B_{B} \quad B_{B}=\frac{2}{r_{o}} \sqrt{\frac{2 I_{b}}{\eta c \beta_{o}}} \quad \sigma=\frac{1-\lambda^{2} \beta_{o}^{2}}{\lambda p^{2}}
\end{gathered}
$$


Setting $r=r_{o} y$ leads to a normalized second order ODE for the beam envelope radius (6). The beam factor $\sigma$ incorporates the beam self-generated fields. The factor $\mu$ is half the cyclotron frequency divided by the speed of light, $\mu=\frac{1}{2} \omega_{c} / c$, and converts time to radian phase $s=$ $\mu \tau=\frac{1}{2} \omega_{c} t$. In the absence of $E_{A},(6)$ is an autonomous ODE [8].

$$
\begin{gathered}
\frac{d^{2} y}{d s^{2}}=-y+\frac{1}{y^{3}}+\frac{\sigma}{y}+\frac{\eta}{r_{o} \mu^{2}} E_{A} \\
y=\frac{r}{r_{o}} \quad s=\mu \tau
\end{gathered}
$$

In the special (single particle) case $\sigma=0$ and $E_{A}=0$, the envelope ODE exhibits the analytic solution ${ }^{1}$ shown in (7). This is an off-axis cyclotron orbit where $y_{c}$ is the orbit center radius and $\epsilon$ is the cyclotron radius. This solution is shown for comparison in Fig. 2 (dashed blue curve). The initial conditions are defined at the emission radius, $r(0)=r_{o}, \beta_{\theta}(0)=0$ and $y^{\prime}(0)$ as given by (9). This solution will serve as a benchmark for understanding the full ODE solutions.

$$
\begin{gathered}
y(s)=\sqrt{y_{c}^{2}+\epsilon^{2}+2 \epsilon y_{c} \sin (2 s-\phi)} \\
y_{o}=y(0)=1 \quad y_{c}=\sqrt{1+\epsilon^{2}} \\
y_{o}^{\prime}=y^{\prime}(0)= \pm 2 \epsilon \quad \phi=\arcsin \left(\frac{\epsilon}{\sqrt{1+\epsilon^{2}}}\right)
\end{gathered}
$$

In the absence of an applied electric field, setting $y^{\prime \prime}=0$ in (6) yields a solvable $4 t h$-order polynomial in $y$. The physically meaningful solution, shown in (10), is termed the equilibrium radius $y_{e q}$. Obviously it is the radius at which $y^{\prime \prime}=0$, but it will be shown that it is the only radius where a ray emitted at $r_{o}$ with beam factor $\sigma$ can have arbitrarily small, including zero, ripple amplitude. This result will prove useful in analyzing the parametric and simulation results.

$$
y_{e q}=\sqrt{\frac{\sigma+\sqrt{4+\sigma^{2}}}{2}}
$$

Setting $E_{A}=0$, the first integral of (6) can be performed analytically as shown in (11). This expression relates the radial position and its first derivative to the ODE initial conditions. The integral of (11) does not have a simple

\footnotetext{
${ }^{1}$ The general solution is $y(s)=\sqrt{\cosh (\kappa)+\sinh (\kappa) \sin (2 s+\phi)}$ where $\kappa$ and $\phi$ are constants, see [8] p296. Equation (7) is this solution specialized to the listed initial conditions.
}

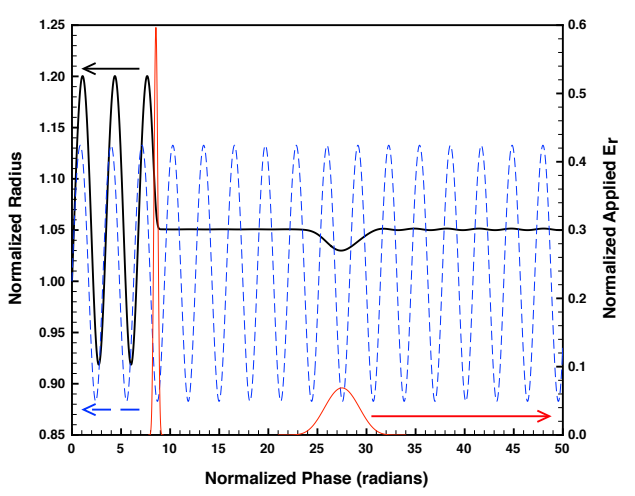

Fig. 2. The solid black curve shows a numerical solution to the envelope ODE model with $\sigma \simeq 0.198, y_{o}=1$ and $y_{o}^{\prime}=0.25$. The orbit initially has sinusoidal ripples with a peak of $y=1.2$ and a period of 3.292 radians. The first radial $E_{A}$ perturbation, lefthand red spike, is non-adiabatic and eliminates the ripple. The second $E_{A}$ perturbation, center red curve, is adiabatic and has almost no lasting effect on the ripple. The dashed-blue curve is (7) with the same initial conditions. Note the differences in ripple amplitude, average radius and period between the special and full solutions.

analytic solution and must be computed numerically. There is no benefit to this as it yields $s(y)$ and only gets more complicated when $E_{A} \neq 0$. Hence, (6) was investigated using a standard ODE solver.

$$
y^{\prime 2}=y_{o}^{\prime 2}+y_{o}^{2}+1 / y_{o}^{2}-y^{2}-1 / y^{2}+2 \sigma \ln \left(\frac{y}{y_{o}}\right)
$$

Equation (11) can provide additional information by noting that $y^{\prime}=0$ at the peaks $\left(y_{p} \geq y_{o}\right)$ and valleys $\left(y_{v} \leq y_{o}\right)$ of the ripples. This results in (12) where $y_{o}^{\prime}$ has been isolated on the left and the square root has been taken. Given $y_{o}$, it is instructive to plot $y_{o}^{\prime}$ as a function of $y_{p / v}$. When plotted, there is an interval of $y_{p / v}$ values where $y_{o}^{\prime}$ has no real solutions. In other words, for a given $y_{o}$, there is a minimum ripple amplitude even if $y_{o}^{\prime}=0$. The only radius at which this is not true is $y_{o}=y_{e q}$ where $y^{\prime \prime}=0$ and $y_{o}^{\prime}=0$ yields zero ripple amplitude.

$$
y_{o}^{\prime}= \pm \sqrt{y_{p / v}^{2}+\frac{1}{y_{p / v}^{2}}-y_{o}^{2}-\frac{1}{y_{o}^{2}}-2 \sigma \ln \left(\frac{y_{p / v}}{y_{o}}\right)}
$$

At $y_{\text {eq }}$, the ripple amplitude has an approximately linear dependance on $\left|y_{o}^{\prime}\right|$. This is demonstrated in (13) where $y_{o}=y_{e q}$ and we set $y_{p / v}=y_{e q}(1+\delta)$ in (12). The expressions inside the square root are expanded in powers of $\delta$ and terms up to $\delta^{3}$ kept. The result is similar 
to the single particle result (9). However, note the $\delta$ dependent asymmetry between peaks, $\delta>0$, and valleys, $\delta<0$.

$$
y_{o}^{\prime} \simeq \pm \sqrt{2}|\delta| \sqrt{y_{e q}^{2}+\frac{1}{y_{e q}^{2}}\left(1-\frac{5+y_{e q}^{4}}{3} \delta\right)}
$$

The behavior of interest is the beam envelope when perturbed by a radial electric field $E_{A}$. In the simulations described in the next section, the radial electric field was produced by the combination of cathode geometry, space charge and the anode aperture as shown by the solidblack curve in Fig. 3B. In Fig. 3A, the aperture component of the field (red dash-dot curve) was extracted from the differences in $E_{r}(z)$ between low-current diode simulations with and without a beam tunnel, dashed-black and solid-black curves respectively. It was determined that this aperture field could be adequately modeled using (14) where $E_{o}$ is the peak amplitude, $s_{p}$ is the phase at which the peak occurs and $2 w$ is the phase width over which the field is non-zero. The analytic and computed aperture fields are compared in Fig. 3A (blue circles and red dash-dot curve respectively) where the analytic model has $E_{o}=-10.45$ statsVolts $/ \mathrm{cm}$, $S_{p}=159$ cells and $w=160$ cells. Figure 3B compares the radial electric field from the full simulation with the computed aperture field combined with the laminar-beam radial self-field. The cathode geometry and space charge significantly alter the radial field in the diode region. Hence, while (14) provides a reasonable means to study beam ripple perturbations, it is clearly a simplification of the actual process.

$$
E_{A}= \begin{cases}E_{o}\left[1-\frac{\left(s-s_{p}\right)^{2}}{w^{2}}\right]^{7} & \frac{\left(s-s_{p}\right)^{2}}{w^{2}} \leq 1 \\ 0 & \frac{\left(s-s_{p}\right)^{2}}{w^{2}}>1\end{cases}
$$

The full ODE (6) with the analytic model for $E_{A}$ was programmed in Mathematica [9] and solved numerically. The parameters ${ }^{2}$ used for this calculation correspond to those in the Dome-1 simulation discussed in the next section, see Table I. The ODE initial conditions were $y_{o}=1$ and $y_{o}^{\prime}=0.25$. The first derivative was calculated using (12) based on the edge ray trajectory peak of $y_{p} \simeq 1.2$ in the simulation. First, examine the two sinusoidal curves on the lefthand side of the figure. These ODE solutions use the same initial conditions but with $\sigma=0.198$ (black) and $\sigma=0$ (dashed blue). Although the curves are of the same character, note the shifts in amplitude, $y_{p}=1.2$ versus $y_{p}=1.13$, average

\footnotetext{
${ }^{2}$ Values for magnetic field, beam current, initial radius, initial peak ripple amplitude, $\gamma_{o}$ and $\beta_{z}$ were taken from the Dome- 1 simulation.
}
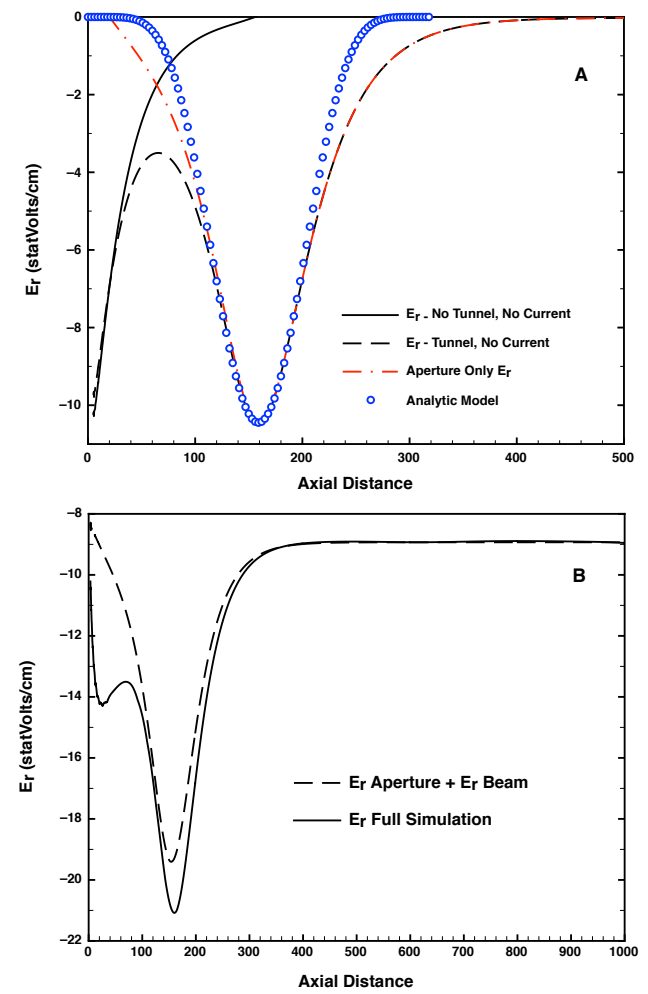

Fig. 3. A: Analytic model for the radial electric field occurring in the beam tunnel entrance in gun simulations. The analytic model is derived from the difference between the radial fields experienced by the edge ray in two low-current $(<26 \mathrm{~mA})$ simulations, one with and one without a beam tunnel. B: Comparison of the radial field from a full $(\sim 26$ A) simulation with the combination of the aperture model and laminar-beam radial field. The horizontal axis is in cell units $h=0.025 \mathrm{~cm} / \mathrm{cell}$.

radius, $\bar{y}=1.059$ versus $\bar{y}=1.008$, and wavelength, $\lambda_{c}=3.292$ radians $\left(\simeq 469\right.$ cells) versus $\lambda_{c}=\pi$ radians ( $\simeq 448$ cells). The beam fields significantly alter the ripple solution and the average radius.

Now focus on the red curve on the lefthand side in Fig. 2. This curve used (14) to model the radial electric field experienced by the beam as it transitions from the diode into the beam tunnel. The position and amplitude of this $E_{A}$ peak were adjusted until the ripple in the solid-black curve was eliminated. Its peak is $E_{o}=22.4 \mathrm{stat}$ Volts $/ \mathrm{cm}$ (plotted values are normalized) and the full width is 1.5 radians. The width of the high-field portion of $E_{A}$ is significantly less than the ripple wavelength of 3.292 radians (black curve). Hence, the lefthand applied field is non-adiabatic relative to the ripple period.

The second component of $E_{A}$, center red curve, exhibits the same integrated area as the first but with 8.67 times the width, spanning almost four ripple periods. This second $E_{A}$ component is adiabatic relative to the ripple period. The different effects caused by these two types of 
perturbation are self-evident in the black curve in Fig. 2 . Before the first perturbation, the cyclotron ripple radius ${ }^{3}$ is 7.17 cells and after it is 0.004 cells. In addition, the constant radius after the first perturbation is equal to the equilibrium radius $y_{e q}=1.0507$ (53.5 cells). The nonadiabatic field causes a permanent change in the orbit, while the effects of the adiabatic field mostly average out. Note that the first $E_{A}$ peak is applied as the orbit approaches $y_{e q}$ on its downward swing and that the transition from rippled to constant radius occurs quickly and smoothly.

\section{Dome CAThode Diode 2D Simulations}

For the diode region, the calculation of beam properties and trajectories is more complex than in the beam tunnel. Further, the transition between the diode and beam tunnel produces radial and axial electric fields that depend on both geometric and beam factors. The dome cathode itself adds complications because aspects of the crossfield emission depend on the dome radius and extension into the anode-cathode (A-K) gap. Hence, self-consistent simulations were used to examine the effects of geometry and guide magnetic field on beam properties.

The simulations used the $2 \mathrm{D}$ electron gun code SCRIBe [10] to calculate diode and beam properties. The calculations consist of an iterative loop involving 1) solution of the Poisson equation, 2) initialization of current-carrying rays at the emission surface, 3 ) propagation of the rays, 4) deposition of space charge and current and return to the Poisson calculation. This loop is repeated until specified convergence criteria are met.

The geometry consists of parallel circular plates with the dome cathode and anode-aperture/beam-tunnel centered on the cylindrical axis, cf. Figs. 1 and 4. The cathode, plates and the beam-tunnel wall are treated as perfect electric conductors. The gap between the plates at the top of the simulation and the radial wall at the end of the beam tunnel are treated as Neumann boundaries. The cylindrical axis is a special symmetry boundary. The simulation parameters for three specific diode geometries are presented in Table I. A square numerical grid of $h=0.025 \mathrm{~cm} /$ cell was used producing a $320 \times 1000$ cell mesh. This section will focus on the Dome-1 simulation results.

The Dome-1 simulation is shown in Fig. 4A and produced the lowest edge ripple observed in this study. The beam consists of 275 rays emitted perpendicular to the dome cathode surface and at an angle to the uniform axial magnetic field. This should result in a

\footnotetext{
${ }^{3}$ Numerical cell units are used to facilitate comparison with the simulation results in the next section.
}

TABLE I

Diode Simulation Parameters

\begin{tabular}{|l|c|c|c|}
\cline { 2 - 4 } \multicolumn{1}{c|}{} & Dome-1 & Dome-2 & Cone \\
\hline Voltage & $60 \mathrm{kV}$ & $60 \mathrm{kV}$ & $60 \mathrm{kV}$ \\
\hline Magnetic Field & $473 \mathrm{G}$ & $473 \mathrm{G}$ & $473 \mathrm{G}$ \\
\hline Diode Radial Height & $8 \mathrm{~cm}$ & $8 \mathrm{~cm}$ & $8 \mathrm{~cm}$ \\
\hline Dome Spherical Radius & $2.2 \mathrm{~cm}$ & $4.0 \mathrm{~cm}$ & - \\
\hline Cathode Face Intercept & $1.28 \mathrm{~cm}$ & $1.25 \mathrm{~cm}$ & $1.28 \mathrm{~cm}$ \\
\hline A-K Gap & $3.9 \mathrm{~cm}$ & $4.1 \mathrm{~cm}$ & $4.0 \mathrm{~cm}$ \\
\hline Beam Tunnel Radius & $2.5 \mathrm{~cm}$ & $2.7 \mathrm{~cm}$ & $2.6 \mathrm{~cm}$ \\
\hline Cone End Point $-Z$ & - & - & $0.22 \mathrm{~cm}$ \\
\hline Cone End Point $-R$ & - & - & $0.19 \mathrm{~cm}$ \\
\hline Beam Current & $26.2 \mathrm{~A}$ & $19.1 \mathrm{~A}$ & $17.7 \mathrm{~A}$ \\
\hline Beam Edge Radius & $1.34 \mathrm{~cm}$ & $1.29 \mathrm{~cm}$ & $1.20 \mathrm{~cm}$ \\
\hline Beam Edge Ripple & $0.2 \%$ & $3.3 \%$ & $2.4 \%$ \\
\hline
\end{tabular}

beam with sizable cyclotron ripples, $\Delta r / r>20 \%$. However, as the beam approaches the anode aperture, the downward cyclotron motion is deflected upward and the rays seemingly become horizontal lines. This can be seen more clearly in Fig. 4B which shows a magnified view of the diode region.
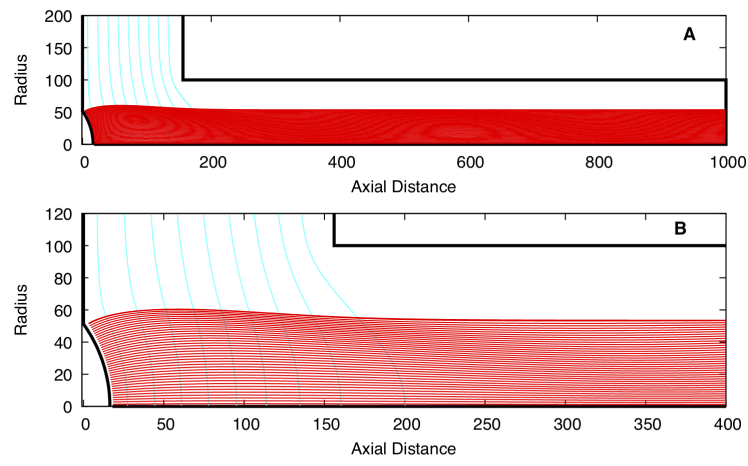

Fig. 4. A: SCRIBe simulation of a dome cathode diode on a $320 \times$ 1000 square grid with $h=0.025 \mathrm{~cm}$. Heavy black lines define the simulation boundaries (full radial extent of 320 cells not shown). The cyan lines are equipotentials. A total of 275 current rays (red lines) were used but only $15 \%$ of the rays were plotted. B: Magnified view of the cathode region in A. Axes are in cell units $h=0.025 \mathrm{~cm} / \mathrm{cell}$.

Other beam characteristics of interest are shown in Fig. 5. Figure 5A shows the beam current density profile at the tunnel exit. The profile is flatter than might be expected from a dome cathode but is not as flat as the beam from a well designed Pierce gun. Figure 5B shows the radial variation in the beam energy and axial velocity. These variations are not large but details of their shape, along with other factors, hint at issues that will be examined in more detail below.

The beam appears straight and laminar in the beam tunnel in Fig. 4, $z \geq 160$ cells. That this is indeed the case is shown by the radial and azimuthal velocity curves in Fig. 6. Both curves appear sinusoidal initially but become horizontal with small oscillations as the beam transitions into the beam tunnel. The radial velocity is 

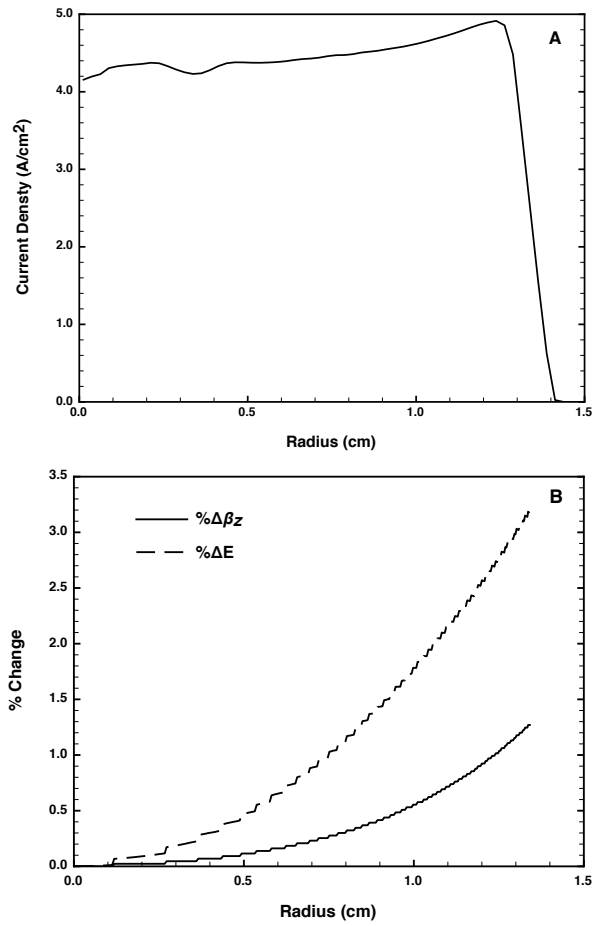

Fig. 5. A: Beam current density versus radius at the beam tunnel exit. A five-point moving average was used to smooth the profile. B: Variation in beam energy and axial velocity versus radius at the beam tunnel exit. Deviations from ideal Pierce-gun radial energy/velocity profiles were noted.

nearly zero and the azimuthal velocity is nearly constant at $\beta_{\theta} \simeq 0.016$. Both curves show a significant change in beam characteristics after the anode aperture transition.

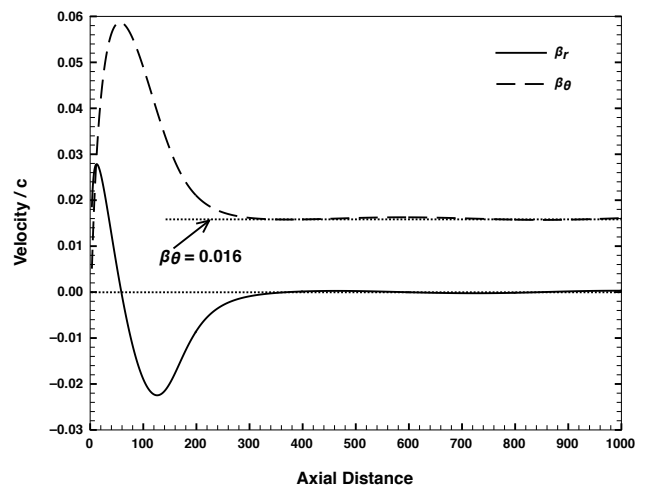

Fig. 6. Radial (solid) and azimuthal (dashed) velocities for the edge ray in Fig. 4. In the beam tunnel, $z>160$ cells, the radial velocity oscillates about zero. The azimuthal velocity has a small oscillation about the beam's bulk azimuthal rotation of $\beta_{\theta} \simeq 0.016$. The horizontal axis is in cell units, $h=0.025 \mathrm{~cm} / \mathrm{cell}$.

Figure 7 plots the edge ray radius along with the radial electric field it experiences. As the ray transits the diode the radius first increases, then peaks and decreases and finally flattens as it enters the beam tunnel. The radial electric field near the dome cathode drives the initial increase in radius. As the ray velocity increases, the $\mathbf{v} \times \mathbf{B}$ force from the uniform magnetic field causes the radius to peak and then decrease. As the ray enters the beam tunnel, it experiences the spike in $E_{r}$ due to both space-charge and anode aperture effects. This spike occurs over an axial span that is significantly less than one ripple period. In the beam tunnel, the ray radius flattens out and the radial electric field reduces to the beam self-field, roughly constant at $E_{r} \simeq-8.9$ statVolts/cm.

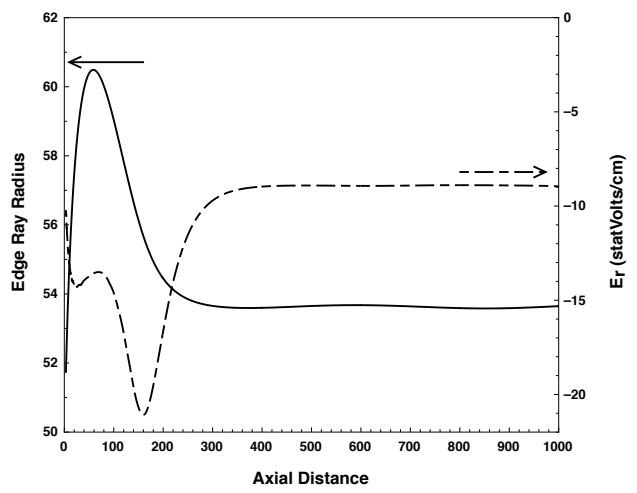

Fig. 7. Overlay of the edge ray trajectory radius and radial electric field from the simulation shown in Fig. 4. The $E_{r}$ spike occurs at the anode aperture as the ray approaches the equilibrium radius, $R_{e q}=53.6$ cells. Axes are in cell units $h=0.025 \mathrm{~cm} /$ cell.

While there are significant differences in the physics included in the simulation versus the ODE calculation, the agreement between the two is surprisingly good. The parameters in the ODE calculation mirrored those in the simulation with some parameters, such as beam current and energy, taken from the simulation. The equilibrium radius in the ODE calculation was 53.5 cells, in excellent agreement with the simulation value of 53.6 cells for the average envelope radius in the beam tunnel, see Fig. 7. The azimuthal velocity at the equilibrium radius calculated from the ODE was $\beta_{\theta}=0.0157$ in good agreement with the simulation value $\beta_{\theta}=0.016$. The ripple wavelengths in the simulation and the ODE calculation were both about 470 cells. Note that the wavelength is modified by the beam self-fields so there is more to this than using the same magnetic field in both computations. The magnitude of the electric field spike needed to eliminate ripple was $22.4 \mathrm{stat} V o l t s / \mathrm{cm}$ in the ODE calculation and about $21 \mathrm{statVolts} / \mathrm{cm}$ in the simulation.

It is worth emphasizing that the transition from rippled 
to flat shown in Fig. 7 exhibits exactly the behavior seen with the ODE solution in Fig. 2. In both cases, a nonadiabatic spike in the radial electric field significantly reduces the ripple amplitudes. And in both cases, the greatest reduction is obtained when the spike occurs on the downward swing of the trajectory before the equilibrium radius is reached. Also note that in both the ODE solution and, surprisingly, in the more complex diode simulation, the transition from an oscillating to flat trajectory is rapid and smooth. The integrated effect of the radial electric field is to reduce $r^{\prime}$ to zero just as the ray reaches the equilibrium radius where $r^{\prime \prime}$ is zero.

\section{BEAM INTERNAL RiPPLE}

The results above show good agreement between expectations based on the ODE solution and beam characteristics obtained in the simulation. However, the plots in Fig. 5, along with other beam diagnostics, show variations with radius that are not completely inline with confined-flow Pierce-gun beams. Examination of beam characteristics in the tunnel highlighted an issue with large cyclotron ripples internal to the beam. The degree of ripple ${ }^{4}$ versus radius in the Dome- 1 beam is shown in Fig. 8 along with three other cases that will be described below. (The dashed line represents a target upper limit of $5 \%$ on ray ripple.) The Dome-1 beam has the lowest ripple at the beam edge but increasingly large ripples (percentage-wise) toward the axis. Setting aside the issue of what effects internal ripple might have on interaction performance, there are two issues to address. First, the causes of the large internal cyclotron motion, and second, can these ripples be reduced or eliminated.

Addressing the first issue, let us return to (6) and examine how the ODE changes at radii internal to the beam. To obtain the same solution, the ODE initial conditions must be the same at each radius. This is easy to do for $y_{o}$ by starting at the emission radius where $y_{o}=1$ by definition. However, $y_{o}^{\prime}$ presents a problem because it is proportional to the radial electric field at the emission surface, which is not constant in magnitude.

Also, changes in the applied field $E_{A}$ versus radius could be part of the problem. In the Dome-1 simulation, the radial electric field at the beam tunnel aperture was found to scale linearly with radius in the beam, i.e. $\left(r / r_{\text {edge }}\right) E_{\text {edge }}$, which yields (15) below. If the ratio $r / r_{o}$ is roughly constant for each ray then the $E_{A}$ term in the ODE will be close to constant with radius.

$$
\frac{\eta}{r_{o} \mu^{2}} E_{A}(r)=\frac{\eta}{r_{\text {edge }} \mu^{2}} \frac{r}{r_{o}} E_{e d g e}
$$

${ }^{4}$ The degree of ripple for a ray was defined as \%Ripple = $200 \cdot\left(R_{\max }-R_{\min }\right) /\left(R_{\max }+R_{\min }\right)$ for the portion of the ray's trajerctory with $z>300$ cells.

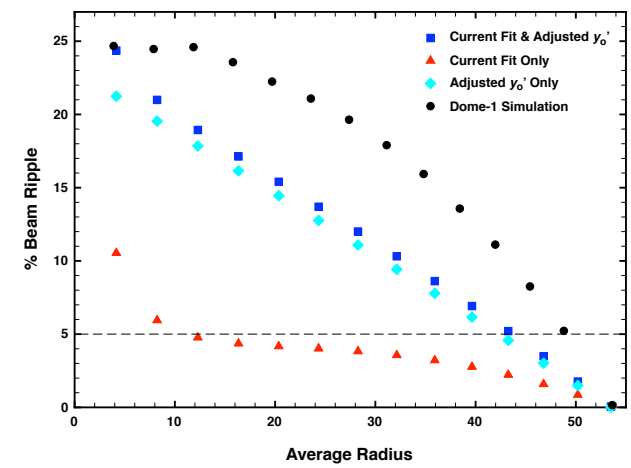

Fig. 8. Internal ripple for the Dome-1 simulation listed in Table I. The Dome-1 cathode has low ripple at the beam edge but sizable internal ripples (black circles). Three ODE model calculations using a $4 \mathrm{th}$ order fit to the Dome-1 enclosed current radial profile (see Fig. 9) are also shown, blue squares, red triangles and cyan diamonds. The dashed horizontal line is at $5 \%$ ripple, the upper limit for acceptable edge ripple. Simulation ripple values were calculated from ray data in the beam tunnel with $z>300$ cells. The horizontal axis is in cell units $h=0.025 \mathrm{~cm} /$ cell.

Factors affecting the ODE normalization constants are also of concern. These include enclosed current $I(r)$, emission radius $r_{o}$, axial velocity $\beta_{z}$, total velocity $\beta$ and $\gamma$. These factors affect the normalization constants $\eta, \mu, p, \lambda$ and $\sigma$. The $\eta$ and $\mu$ constants depend on $\gamma$, which does not change significantly for the Dome-1 beam. The other three constants depend on current, $r_{o}, \beta$ and $\beta_{z}$. The total and axial velocities vary by $\sim 1 \%$ each over the beam radius and their ratio $\lambda$ has a maximum variation of $0.2 \%$. These variations are not sufficient to cause the ripple increase seen in the Dome-1 simulation.

In addition to $y_{o}^{\prime}$, the enclosed current profile is potentially a cause of the internal ripples. Assuming no ray crossings, the current enclosed by a ray for a uniform density beam can be expressed as $I_{\text {enclosed }}\left(r_{o}\right)=$ $\left(r_{o} / r_{o M a x}\right)^{2} I_{o}$. This ideal density profile removes the current and $r_{o}$ dependancies from $p$ and $\sigma$. This ideal profile is compared with the Dome-1 simulation results in Fig. 9. The two profiles do differ, but the question is whether the difference is sufficient to cause the internal ripples.

The roles that the current profile and the initial ray radial velocities $y_{o}^{\prime}$ play were explored using the ODE model. The simulation current profile was fit with a $4 t h$-order polynomial, see Fig. 9, which was programmed into the model. Then three cases were run: 1) Simulation current profile with $y_{o}^{\prime}$ held at the beam edge value, 2) Ideal current profile with $y_{o}^{\prime}$ varied as the sine of the emission angle, and 3) Simulation current profile and $y_{o}^{\prime}$ varied. The results are compared with the Dome-1 ripple profile in Fig. 8. While the non-ideal simulation current profile does contribute to increased ripple, it's clear that the 


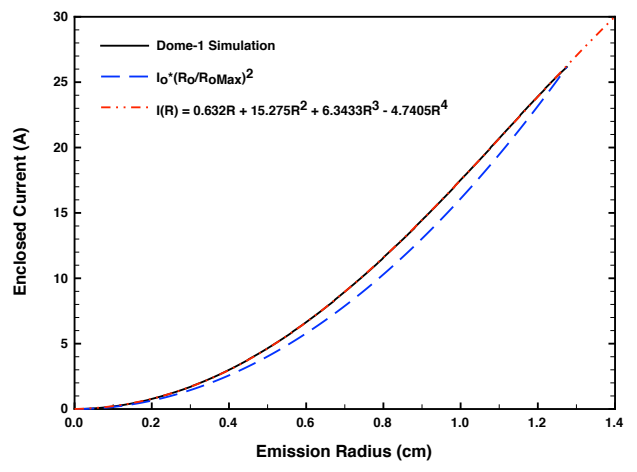

Fig. 9. Enclosed current profile for the Dome-1 beam (solid black curve). Also shown are the ideal enclosed current profile (dashed blue curve) and a $4 t h$-order polynomial fit to the simulation data (dash-dot red curve).

mismatch in $y_{o}^{\prime}$ was the primary driver of internal ripples in the Dome-1 simulation.

Two approaches were taken in attempting to reduce internal ripple. The first was variation of the Dome-1 parameters with reduced emphasis on the edge ripple. The second was a cone cathode geometry with a roughly constant emission angle, also with reduced emphasis on edge ripple. The parameters for both cathodes are given in Table I in the Dome- 2 and Cone columns respectively. The emission surfaces for the Dome-1 (A), Dome-2 (B) and Cone $(\mathbf{C})$ simulations are shown in Fig. 10. The plots show that both of the new configurations provide more uniform emission angles, the cone by design and the dome by use of a larger spherical radius. Several factors resulted in significantly lower beam currents in the new designs.
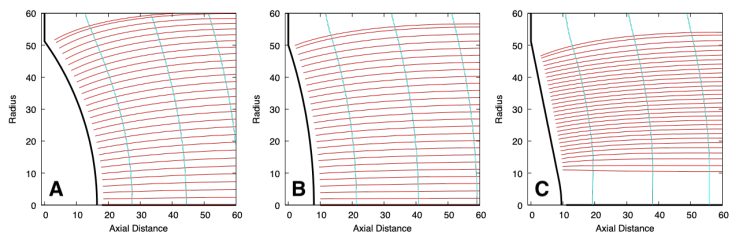

Fig. 10. Emission surfaces for the three cathodes in Table I; A: Dome1, B: Dome-2 and C: Cone. Only about $10 \%$ of the rays used in the simulations are plotted with the first and last rays always included. The axes are in cell units $h=0.025 \mathrm{~cm} /$ cell.

The internal ripple profiles for the two new cathodes are shown in Fig. 11. The Dome-2 beam is well under the $5 \%$ ripple limit except near the axis. The Cone beam has lower edge ripple and remains under the $5 \%$ ripple limit until the inner edge of the beam. Both of these beams should perform well for a variety of interaction circuits. Both simulated designs are "basic" in the sense that no sculpting of the diode surfaces was done.

The performance shown in Fig. 11 should be considered

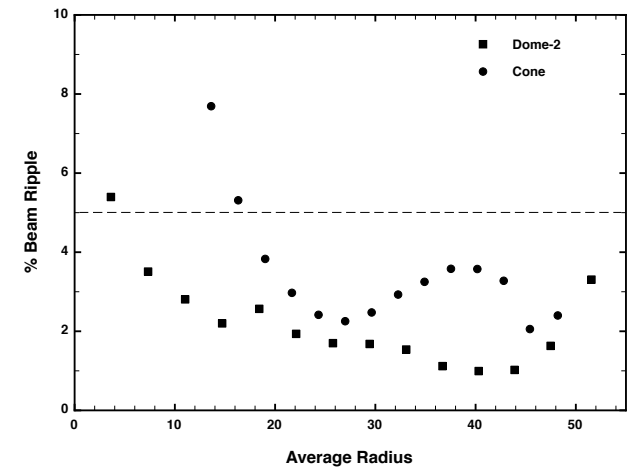

Fig. 11. Internal beam ripple results from simulations of the alternate dome and cone cathode geometries, see the Dome- 2 and Cone columns in Table I. In both cases, the majority of the beam is well below the $5 \%$ ripple limit. Both cathode geometries have issues with increased ripple at the inner and outer beam edges. The horizontal axis is in cell units $h=0.025 \mathrm{~cm} /$ cell.

the minimum obtainable by these configurations. Cone cathode performance can be improved by shaping of the cone surface at the inner and outer edges of the emission region. For the dome cathode, performance might be improved by using a shape differing slightly from spherical, perhaps elliptical. Additional methods, some discussed below, are available to mitigate internal beam ripple. So this aspect of dome-type diodes can be controlled.

\section{Robustness AND FleXibILITY}

It was demonstrated above that a non-adiabatic radial electric field spike of appropriate amplitude, delivered at the correct cyclotron phase, can reduce or eliminate beam ripple. Hence, variations in parameters that affect the cyclotron wavelength (voltage, axial magnetic field and beam current), the $E_{r}$ spike (A-K gap, tunnel radius and beam current) and emission conditions (spherical radius, cone length/angle and cathode wall intercept) can negatively impact ripple throughout the beam.

To examine design sensitivity, six diode parameters were individually varied by $\pm 3 \%$ and a simulation run with each varied value. Note, this was not an optimization process but an assessment of how well centered the diode configurations were relative to edge ripple. The "spider" plots shown in Fig. 12 illustrate the variation effects on beam ripple for the three Table I configurations.

The plots indicate that the modified geometries exhibit a more centered response to variation, albeit at the cost of higher edge ripple. Edge ripple remained below $5 \%$ for all variations but two, the Dome-2 responses to increases in the magnetic field and A-K gap. Indeed, all three configurations exhibited sensitivity to A-K gap 

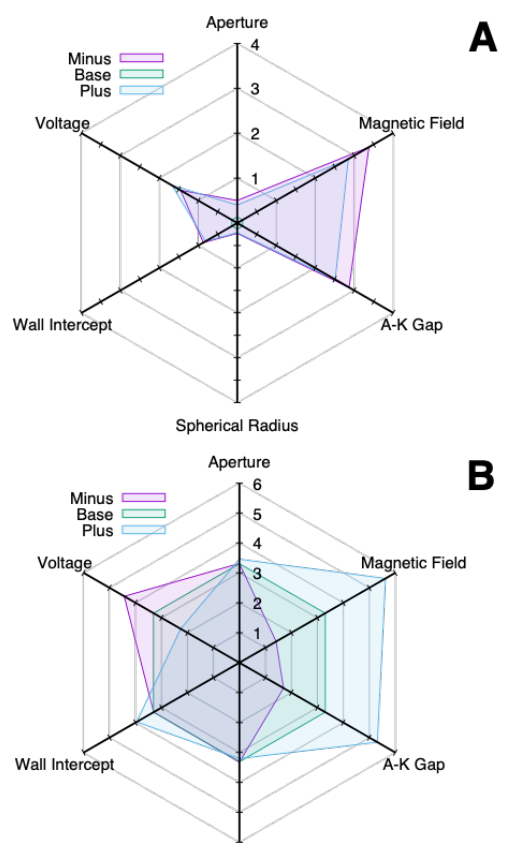

B

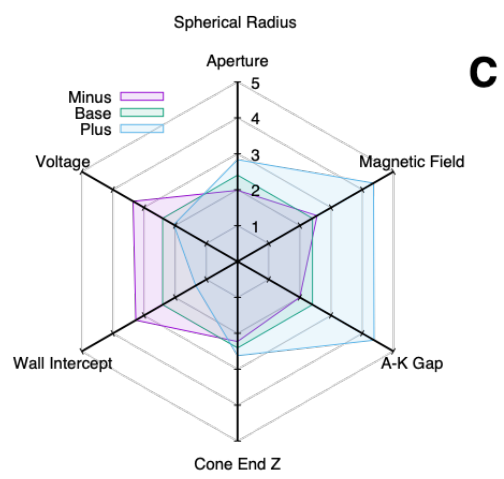

Fig. 12. Changes in beam edge ripple for the Dome-1 (A), Dome-2 (B) and Cone $(\mathbf{C})$ diodes. Each axis shows the \%-ripple of the beam edge ray with $-3 \%$ (red) or $+3 \%$ (blue) variation of that parameter. The base $\%$-ripple from Table I is shown in green. The varied parameters are (clockwise from top) 1) anode aperture radius, 2) axial magnetic field, 3) A-K gap, 4) spherical radius/cone end-point z-value, 5) cathode wall intercept radius and 6) diode voltage.

and magnetic field variation. These two factors directly affect the alignment of the ray trajectory with the radial electric impulse, a key element in reducing ripple. Since there was nothing special about the chosen simulation parameters, we take this as an indication of general robustness for the dome/cone cathode configurations.

The three cathode configurations examined above indicate a reasonable degree of design flexibility for dometype diodes. However, design always involves tradeoffs in order to meet overall requirements. The need to align the trajectory phase and the $E_{r}$ spike links the voltage, magnetic field and the A-K gap. While this can pose challenges, parameters and geometry can be varied to work around resulting limitations. For example, the cone

diode in Table I could be suitable for some application except a 20 A beam is needed. By simultaneously shrinking the A-K gap $(3.6 \mathrm{~cm})$ and increasing the magnetic field $(522 \mathrm{G})$, beam current was increased to 20.26 A. The ripple profile was essentially the same as before, see Fig. 13. Other techniques such as shaping electrode surfaces and adding electrodes to alter/control field shapes are common in beam diode design.

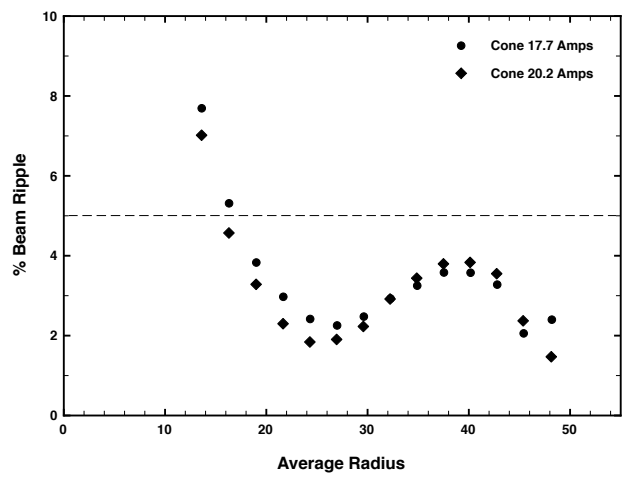

Fig. 13. Comparison of beam ripple profiles from the same cone cathode with different A-K gaps and axial magnetic fields. Horizontal axis is in cell units $h=0.025 \mathrm{~cm} /$ cell.

Additional flexibility can be obtained post-diode by adiabatic and non-adiabatic changes in the beam tunnel radius. This is illustrated in Fig. 14 where simulations with an adiabatic taper in tunnel radius (A) and a nonadiabatic step in radius (B) are shown. Beam edge radii for these two cases are compared with the Dome-1 uniform tunnel result in Fig. 15. The taper has almost no effect on edge ripple and the step produces only a slight increase. Step changes, if executed at the right cyclotron phase, could reduce beam ripple in the same manner as the anode aperture.
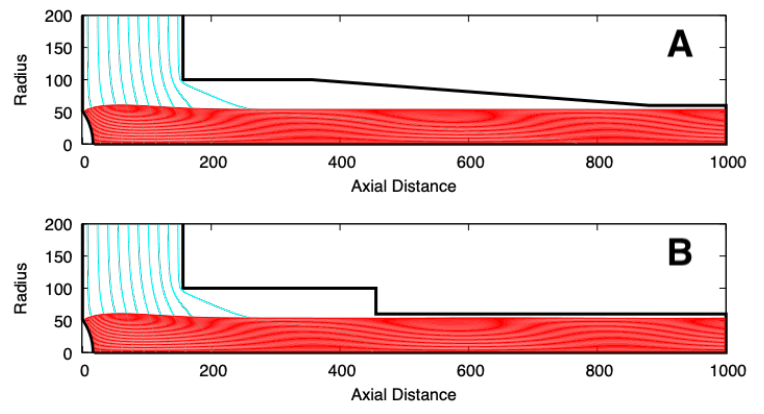

Fig. 14. Two possible post-diode beam tunnel profiles. A: A gradual taper in the beam tunnel radius from the $2.5 \mathrm{~cm}$ anode aperture down to a $1.5 \mathrm{~cm}$ exit radius. $\mathbf{B}$ : A sharp step down to the $1.5 \mathrm{~cm}$ exit radius. All other parameters are the same as the Dome-1 uniform tunnel radius simulation in Fig. 4A. Axes are in cell units $h=0.025 \mathrm{~cm} / \mathrm{cell}$. 


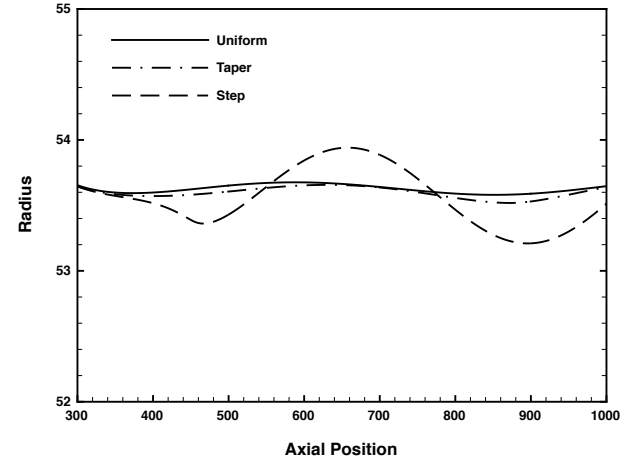

Fig. 15. Effects of beam tunnel radial profiles on edge ripple compared to the uniform radius result. The tunnel radial profiles are shown in Fig. 14. The gradual taper produces a beam ripple very close to that of the uniform radius tunnel. The step-change profile increases beam ripple more than the taper but the change is not large. Axes are in cell units $h=0.025 \mathrm{~cm} / \mathrm{cell}$.

\section{SUMmary AND CONCLUSIONS}

In retrospect, there were two serendipitous accidents in the original research that prompted this study. The first was the convex spherical (dome) shape of the cathode and the second was the lack of Pierce-type anode focusing structures. The first was a case of the NCSU students not knowing their choice was "impossible." The second was a deliberate choice made to simplify the optimization procedure for the students. Combined, these departures from the norm showed that a new electron gun configuration could unexpectedly produce beams with extremely low envelope ripple.

This research demonstrated the unexpected synergistic effects of using a non-adiabatic $E_{r}$ impulse to control beam ripple. The physics underlying this effect was illuminated through analytic model calculations and 2D simulations. Analytically, it was shown that each ray has an equilibrium radius determined by its emission point and beam factor. Only at this radius is it possible for the ray to have zero ripple. By properly aligning the phase of the beam cyclotron orbit and the abrupt transition at the anode aperture, it is possible to achieve near total elimination of beam envelope ripple. This was shown to occur at the equilibrium radius of the beam envelope using 2D simulations. Although general in nature, this principle works well in diodes immersed in uniform magnetic fields. This has important practical benefits due to reduced sensitivities to alignment tolerances and less complex focusing structures.

A potential problem with diodes using dome cathodes was identified, beam internal ripple. The causes of inter- nal ripple were pinpointed, mainly emission details with a smaller contribution from a non-ideal enclosed-current profile. It was demonstrated with two cathode geometries that internal ripple is controllable. Additional control should be possible with the inclusion of focusing structures. Note that designing toward an optimum profile requires assessment of the impact of internal beam ripple on interaction performance. This will likely require a different quality metric than the percent ripple used in this work.

The dome-cathode effect was shown to be robust through parameter variation simulations. Further, the effect was shown to work with a different geometry, i.e. the cone cathode. Knowing the physical basis of the effect enables flexible reconfiguration of parameters and geometry to meet design needs. The dome-cathode effect can be utilized in multi-beam and distributed-beam configurations without increasing interaction between the beams. Because of difficulties in controlling emitting surfaces, diodes in high-voltage, pulsed-power devices might find the dome-cathode effect especially useful. Non-intercepting shaping of the anode aperture and postdiode steps in wall radius could be configured to reduce (or enhance) beam cyclotron motion.

In addition, we note that the dome-cathode effect will work for concave (standard) cathodes immersed in uniform magnetic fields. Although, since the initial cyclotron motion in such geometries in downward, higher magnetic fields and/or larger anode-cathode gaps will be required.

As mentioned in the introduction, two recent papers [11], [12] have examined the use of non-adiabatic electric and magnetic fields in electron gun diodes, with experimental results reported in [11]. These papers were primarily concerned with electron guns for applications in highenergy accelerator physics and focused more on use of a magnetic impulse. While the ability to tune a magnetic impulse is beneficial in experimental research, it is not ideal for deployed devices. Additionally, magnetic impulses are not compatible with confined-flow distributed beam configurations because of the difficulty in shielding multiple magnetic impulses from each other and the resulting interference. In contrast, a nonadiabatic radial electric impulse is an inherent aspect of the diode geometry and is easy to shield in distributedbeam configurations.

The detailed understanding of the "dome-cathode effect" developed in this paper permits simplification of both beam diode geometry and magnetic field profile/alignment. Improvements in reliability, scalability and mechanical alignment/tolerance requirements (hence, lower costs) follow directly from this. Both 
alternate approaches to laminar beam generation, electric and magnetic impulse, will benefit research on advanced performance RF devices.

\section{REFERENCES}

[1] "Computational Design of Asymmetric Electron Beam Devices", R. L. Ives et al., IEEE Transactions on Electron Devices, 56, No. 5, pp. 753-761 (2009), doi: 10.1109/TED.2009.2015421.

[2] "Controlled Porosity Cathodes for High Current Density Applications", R. Lawrence Ives, Louis R. Falce, George Miram, George Collins, IEEE Trans. Plasma Sci., Special Edition on High Power Microwave Sources, 38, No. 6, pp. 1345-1353, June 2010.

[3] "A review on scandia doped tungsten matrix scandate cathode", Wang, J., Yang, Y., Wang, Y. et al., Tungsten 1, pp. 91-100 (2019), doi: 10.1007/s42864-019-00007-8.

[4] Modern Developments in Vacuum Electron Sources, Topics in Applied Physics 135, George Gaertner, Wolfram Knapp, Richard G. Forbes, Editors, Springer, ISBN 978-3-030-47290-0, 2020.

[5] "A $200 \mathrm{MHz} 35 \mathrm{MW}$ Multiple Beam Klystron for Accelerator Applications," Final Report, U.S. Department of Energy Grant No. DE-SC0000804, August 24, 2010.

[6] Information on the CCR Beam Optics Analyzer (BOA) code can be found at https://calcreek.com/products/software/beam-opticsanalyzer/

[7] "Low and Zero Compression Electron Guns", Michael Read, Lawrence Ives, Thuc Bui, Hien Tran, 11th IEEE Intern. Vacuum Elect. Conf, Monterey, CA, May 2010.

[8] Handbook of Differential Equations, D Zwillinger, Academic Press, 1989.

[9] Wolfram Research, Inc., Mathematica, Version 11.3, Champaign, IL (2018).

[10] SCRIBe is a highly modified version of W. B. Herrmannfeldt's EGUN code (circa 1977). See "EGUN - An Electron Optics and Gun Design Program", SLAC - Report - 331, 1988.

[11] "Analysis of magnetically immersed electron guns with nonadiabatic fields", Alexander Pikin, James G. Alessi, Edward N. Beebe, Deepak Raparia, and John Ritter, Review of Scientific Instruments 87, 113303 (2016).

[12] "Method of controlling the cyclotron motion of electron beams with a nonadiabatic magnetic field", Alexander Pikin, Hannes Pahl, and Fredrik Wenander, Phy Rev Accelerators and Beams 23, 103502 (2020). 